\title{
Le récit semi-autobiographique comme angle d'approche de la culture des milieux de la finance en anglais de spécialité : l'exemple de Liar's Poker
}

Approaching organizational culture in the world of finance through semiautobiographical narratives: the case of Liar's Poker

\section{Marc Eline}

\section{OpenEdition}

Journals

Édition électronique

URL : http://journals.openedition.org/asp/4979

DOI : 10.4000/asp.4979

ISSN : 2108-6354

\section{Éditeur}

Groupe d'étude et de recherche en anglais de spécialité

Édition imprimée

Date de publication : 1 mars 2017

Pagination : 123-143

ISSN : 1246-8185

Référence électronique

Marc Eline, "Le récit semi-autobiographique comme angle d'approche de la culture des milieux de la finance en anglais de spécialité : l'exemple de Liar's Poker», ASp [En ligne], 71 | 2017, mis en ligne le 01 mars 2018, consulté le 01 novembre 2020. URL : http://journals.openedition.org/asp/4979 ; DOI https://doi.org/10.4000/asp.4979

Ce document a été généré automatiquement le 1 novembre 2020.

Tous droits réservés 


\title{
Le récit semi-autobiographique comme angle d'approche de la culture des milieux de la finance en anglais de spécialité : l'exemple de Liar's Poker
}

\author{
Approaching organizational culture in the world of finance through semi- \\ autobiographical narratives: the case of Liar's Poker
}

Marc Eline

1 Étudier l'anglais de la finance ${ }^{1}$ consiste, comme pour toute variété spécialisée de l'anglais, à aller au-delà de l'approche terminologique qui permet d'appréhender les concepts du domaine, pour élargir le champ d'investigation en s'intéressant au contexte textuel et culturel propre au milieu concerné, sans négliger l'axe diachronique. La prise en compte de la double dimension langue-culture est, en effet, essentielle pour approfondir la compréhension d'un milieu et de sa culture, en ayant appréhendé la façon dont il est structuré et fonctionne ainsi que les règles qui régissent la communication et les relations entre les acteurs de ce milieu spécialisé. Maittriser l'anglais de la finance exige donc d'être au fait de la culture et de la logique professionnelles qui prévalent dans les milieux de la finance.

2 C'est en nous fondant sur cette logique que nous avons envisagé de choisir comme angle d'approche le récit d'un professionnel : dans Liar's Poker', Michael Lewis décrit ses deux ans et demi d'activité chez Salomon Brothers ${ }^{3}$ en tant que vendeur d'obligations à New York et à Londres dans les années 1980. Si notre expérience personnelle nous permet d'aborder ce récit en nous référant à notre propre connaissance et pratique du milieu, nous sommes conscient que la plupart des chercheurs en anglais de spécialité ont eu un itinéraire essentiellement universitaire. Nous avons gardé ce point à l'esprit lors de notre analyse, et nous désirons montrer que l'exploitation de ce type de récit peut également aider le lecteur profane à enrichir sa connaissance du milieu. Étant 
entendu qu'un regard critique s'impose pour faire la part des choses en analysant un témoignage de première main, il convient de prendre en compte la période concernée et les motivations de l'auteur. Dans un premier temps, nous présentons Liar's Poker et son auteur ${ }^{4}$ pour justifier notre choix et souligner l'intérêt que présente cette source d'information. Puis, après un rappel du cadre théorique et de la méthodologie adoptés pour notre analyse, nous présentons les résultats de l'étude et en offrons une mise en perspective et une discussion.

\section{Présentation et intérêt du choix de l'ouvrage}

3 L'ouvrage de Lewis est à la fois un récit autobiographique, un bestiaire des " animaux " qui peuplent le biotope qu'est Wall Street et un recueil d'anecdotes savoureuses sur le comportement des traders, des vendeurs et sur leurs interactions avec leurs interlocuteurs divers, qu'il s'agisse des pouvoirs politiques, des investisseurs, ou des journalistes.

4 Le récit est composé de onze chapitres (298 pages) et suivi d'un glossaire. Le livre est empreint d'un souci didactique récurrent, comme l'atteste la présentation des activités financières assortie d'une description détaillée des produits et montages ${ }^{5}$. L'ouvrage a d'ailleurs reçu un très bon accueil de la critique, dans la presse générale ${ }^{6}$ comme dans les journaux financiers. À titre d'exemple, l'édition de 2006 du livre mentionne de nombreux avis favorables tirés de The Economist, The Financial Times, International Financial Review et de Management Today. Dans le numéro du 20 novembre 1989 de Fortune, le critique voit dans l'ouvrage de Lewis « one of those rare works that encapsulate and define an era» (Kirkland 1989). Un fil conducteur souple permet, par la voix de l'auteur, de suivre son parcours au sein de la banque, de son embauche jusqu'à son départ en 1988. L'occasion lui est ainsi donnée de couvrir les pratiques d'embauche des jeunes diplômés des meilleures universités, leur programme de formation et d'insertion, la description des métiers et de leurs perceptions, le quotidien des équipes opérant sur les marchés et l'évolution de l'activité. Lewis observe notamment les succès impressionnants de Salomon Brothers, puis les bouleversements économiques et financiers qui affectent la banque et son incapacité à faire évoluer son modèle d'affaires ; la conjonction de ces difficultés entraîne inexorablement la banque dans une crise profonde. L'épilogue lui offre un prétexte pour se livrer à quelques réflexions sur les métiers de la finance, la place de l'argent et le bonheur.

5 Le choix d'un récit spécialisé comme source d'informations quant à la culture des milieux de la finance est motivé par l'idée qu'un témoignage de première main d'un professionnel offre une autre perspective que celle proposée par des brochures promotionnelles et autres supports de communication institutionnelle, conçus par les banques avec le concours d'agences de relations publiques. En effet, la communication institutionnelle, telle qu'elle se présente dans des textes comme les mission statements de telle ou telle entreprise, ou dans l'annonce de ses valeurs ou objectifs est souvent trop uniforme (Resche $2005: 16$ ) et policée : de ce fait, elle risque de mettre en avant une culture téléologique ou des déclarations programmatiques bien éloignées de ce qui ressort du témoignage de l'auteur dans son récit.

6 Certes, la presse ou les forums en ligne offrent parfois la possibilité de se représenter le quotidien des professionnels, mais une plongée de plus longue durée dans ces métiers s'impose si l'on veut saisir l'ensemble de la culture d'entreprise ou du secteur, ses 
valeurs profondes, les nuances et les non-dits. Or, la durée d'activité proprement dite de Lewis, qui porte sur la période 1985-1988, est généralement admise dans le milieu comme suffisante pour construire une véritable première expérience professionnelle, valorisable sur le marché7. Nous sommes conscient que la vigilance s'impose toutefois quant aux limites à l'objectivité et à l'impartialité inhérentes au récit: quand bien même l'auteur préciserait qu'il a librement décidé de quitter Salomon Brothers et se défendrait de toute acrimonie, il reconnaît avoir renoncé à une carrière qui s'annonçait prometteuse.

7 Sélectionner le livre de Michael Lewis nous a semblé également justifié par plusieurs facteurs. En premier lieu, Liar's Poker fait désormais partie des ouvrages de référence sur les professionnels du secteur bancaire durant les années 1980 dans les deux grandes places financières de Londres et New York où Lewis a exercé. Ce livre figure encore actuellement dans la liste de lectures imposées ou recommandées dans plusieurs cours universitaires aux États-Unis ${ }^{8}$. Il est mentionné dans des forums internet que consultent de futurs étudiants en business schools ou des étudiants en classes préparatoires aux écoles de commerce françaises dans les années $2010^{\circ}$. De même, Liar's Poker est en bonne place dans la liste des livres fondamentaux traitant de finance ou de management sélectionnés par la presse américaine (The 20 Most Influential Business Books de Forbes, septembre 2002, ou Essential Wall Street Summer Reading List du New York Times, juillet 2013). Dans l'édition du 30 septembre 2002, un journaliste de Forbes Magazine considérait que des récits qui ne sont pas des ouvrages de fiction tels que Barbarians at the Gate (Burrough \& Helyar 1990) et Liar's Poker continuaient d'exercer une très forte influence sur l'image que se fait le public de Wall Street et du monde des affaires. D'autres livres relatifs au milieu de la finance américaine de cette période ont connu un grand succès commercial : Den of Thieves (Stewart 1991), The Predators' Ball (Bruck 1988) ou encore The Bonfire of the Vanities (Wolfe 1987) illustrent les succès de Wall Street, mais aussi ses excès et leurs conséquences. Ils diffèrent cependant du récit semiautobiographique de Liar's Poker par leurs approches, qui privilégient soit l'analyse critique de l'institution Drexel Burnham et du banquier "prédateur " Michael Milken, spécialiste des junk bonds, soit le roman sur fond de délit d'initiés, d'ego surdimensionnés et de tensions raciales et culturelles à New York.

Liar's Poker décrit la vie quotidienne des professionnels de Salomon Brothers, banque d'investissement qui a été à l'origine de diverses innovations financières marquantes et qui fut, à cette époque, un leader reconnu sur la place financière de New York, notamment dans le marché des produits de taux. Salomon Brothers s'était également distinguée durant les années 1960 : elle faisait alors partie, avec des institutions telles que Lehman Bros et Kidder Peabody, d'un groupe surnommé les Fearsome Foursome, en raison de leur agressivité commerciale et de leur prise de risque hors normes sur le marché des émissions de titres (O'Sullivan 2013: 291). Le livre de Lewis souligne combien l'agressivité et l'appétit pour le risque sont toujours au cœur des pratiques de Salomon Brothers, trente ans plus tard.

9 Le terme de « finance " recouvre un large spectre d'activités. Nous ne nous intéressons pas ici aux activités de la banque commerciale, dont le rôle principal est de collecter l'épargne, de gérer les dépôts de ses clients et de financer directement particuliers et entreprises sur la base de ses ressources. Le périmètre de notre étude est celui des activités de marché de capitaux, qui soulèvent la question du risque qu'elles font encourir à l'établissement, mais aussi à ses clients et à ses partenaires, voire 
globalement à l'économie, en cas de faillites en chaîne. À la différence d'autres récits de banquiers à dominante autobiographique ${ }^{10}$, c'est précisément sur ces activités de marché, concentrées au sein des équipes de vente et de trading ${ }^{11}$, que porte Liar's Poker.

La finance américaine des années 1980 est marquée par la conjonction de facteurs de changement qui ont profondément et durablement transformé le monde de la banque et de la finance. Sur le plan scientifique, les banques ont bénéficié des découvertes des décennies précédentes en matière financière ${ }^{12}$, qui ont permis de structurer à grande échelle des produits d'endettement nouveaux et sophistiqués, avec l'aide des ordinateurs et des algorithmes (Chappe et alii 2012:65). Sur le plan réglementaire, la libéralisation au Royaume-Uni (le «Big Bang» thatchérien de 1986) et la déréglementation aux États-Unis ont favorisé l'émergence des banques au détriment des traditionnelles maisons de courtage londoniennes et ont provoqué, dans le cas américain, une forte volatilité des taux, dont les conséquences ont été un mouvement notable d'innovation financière et de spéculation (Abolafia 2001 [1996] : 5). La période 1981-1986 est caractéristique de la financiarisation rapide de l'économie américaine : le volume d'endettement par émission d'obligations garanties par l'État connaît une croissance annuelle de $37 \%$, le montant en dollars des opérations de fusionsacquisitions triple (Cetina \& Preda $2005: 2$ ). Dans un entretien accordé à une société de conseil en $2010^{13}$, Lewis n'hésite pas à voir un lien de causalité entre la mutation brutale qui affecte la finance durant les années 1980 et la crise qui survient près de trente ans plus tard:

[...] what I had actually written was a description of the beginning of a 30-year-long traumatic event. I could see how [the most recent] financial crisis was really the natural conclusion of things I had written about back in 1989.

11 Notre choix a également été guidé par le fait que, à la différence des autres ouvrages du même auteur (cf. bibliographie), dont la matière (finance quantitative moderne, haute technologie, sport, etc.) est généralement issue de milieux dans lesquels Lewis n'a pas nécessairement évolué professionnellement ${ }^{14}$, Liar's Poker est un récit à la première personne d'une expérience directement vécue par l'auteur.

\section{Cadre théorique}

\subsection{Un genre hybride, entre FASP et autobiographie}

Dans Liar's Poker, l'auteur alterne histoire personnelle, avec le récit de son vécu, objet de questionnements progressifs à mesure qu'il prend du recul sur son expérience, et histoire sociale, celle de ses collègues, de ses supérieurs hiérarchiques, de ses clients et des interactions entre ces différents acteurs de la finance. Lewis, conscient de ses faiblesses techniques et de la chance qui lui a permis de décrocher son emploi, n'hésite pas à manier l'autodérision. Il reconnaît par ailleurs avoir participé à des opérations discutables: il a contribué, par exemple, à soulager financièrement la banque au détriment d'un des clients dont il avait la responsabilité. Il est cependant permis de se demander s'il ne cède pas à une certaine tentation hagiographique, lui permettant d'endosser en fin de récit l'habit du repenti conscient de l'inanité de son mode de rémunération, après avoir été victime d'une cécité temporaire due à la "culture Salomon » qui l'aurait privé de ses repères éthiques. 
When you sit [...] at the centre of [...] the most absurd money game ever, and [...] benefit out of all proportion to your value to society [...], what happens to the money belief? [...] For me, the belief in the meaning of making dollars crumbled. d'événements dont l'auteur n'a même pas nécessairement été témoin direct, comme la partie de «poker menteur » entre les deux cadres dirigeants de Salomon Brothers qui donne son titre à l'ouvrage. Denis Collins $(1992: 376)$ rapporte que plusieurs élèves de son cours d'éthique des affaires réagissaient à la lecture de Liar's Poker en demandant si le récit était une histoire vraie. De nombreux épisodes (par exemple, le dîner dans la haute société anglaise ou le bizutage des nouveaux analystes financiers) nous conduisent à comparer l'ouvrage de Lewis à une fiction à substrat professionnel (FASP), genre de récit spécialisé défini comme suit par Michel Petit (2000:173-174) :

Peut être considéré comme ressortissant à la FASP tout texte de fiction commerciale à grand succès (i) relevant généralement du thriller spécialisé [...], (ii) utilisant un milieu professionnel particulier non seulement comme cadre général de l'histoire mais aussi et surtout comme l'une des sources principales des ressorts de l'intrigue, (iii) exprimé dans une langue reproduisant les pratiques langagières [...] de ce milieu, et (iv) généralement écrit par un auteur dont l'appartenance ou les liens avec ce milieu sont explicitement revendiqués.

Lewis est un professionnel des milieux financiers qui, comme les auteurs de FASP, a évolué du statut de professionnel auteur vers celui d'auteur professionnel.

Liar's Poker est-il un "thriller » financier? Les thrillers comportent généralement un dénouement inconnu du lecteur, alors que, dès la préface, Lewis donne à comprendre certes sans en préciser le contexte exact - que son histoire chez Salomon est terminée. Pour autant, le côté captivant du livre, les intrigues relatives aux luttes de pouvoir rappellent certaines caractéristiques des thrillers. De même, la révélation progressive des aspects sombres d'un milieu lié à l'argent, fermé et brutal, objet de fascination de la part du grand public, n'est pas sans évoquer le travail d'investigation auquel procèdent souvent les héros de thrillers. La période des années 1980, décrite comme un nouvel âge d'or (et d'excès) par M. Van der Yeught (2009 : 262-263), se prête particulièrement bien à la production de "thrillers financiers", tant sous une forme littéraire que cinématographique; la décennie est marquée par l'apparition de nouveaux acteurs de la finance qui réalisent des transactions d'un montant astronomique en exploitant l'effet de levier des junk bonds ${ }^{15}$, par l'envolée des salaires des professionnels et tout particulièrement de ceux des dirigeants, par un cynisme ambiant qui voit des prédateurs financiers s'enrichir aux dépens de leurs cibles et par des spéculateurs qui n'hésitent pas à déclarer publiquement que "Greed is all right » ${ }^{16}$. Liar's Poker, comme les titres des ouvrages de FASP financière de cette période ${ }^{17}$, se veut un titre attractif pour un lectorat qui s'interroge sur les infamies dont sont capables les banquiers de l'époque. De même, le paratexte retenu par l'éditeur vient appuyer la démarche marketing commune à la FASP avec, en couverture, un commentaire favorable de Tom Wolf $\mathrm{e}^{18} \mathrm{et}$, en quatrième de couverture, un avis élogieux du Sunday Times. L'auteur de la critique laisse en effet entendre que la réalité dépeinte dans Liar's Poker ne décevra pas les amateurs de Wall Street, devenu entre-temps un film à succès. À certains égards, on peut donc considérer que Liar's Poker possède des caractéristiques typiques de la FASP.

Toutefois, étant donné que l'auteur-narrateur, parfaitement identifié, écrit à la première personne un récit portant sur une partie de sa vie professionnelle, dans une perspective principalement rétrospective, il s'ensuit que le livre relève formellement 
de l'autobiographie. Pour autant, le récit sort du cadre strictement autobiographique quand le futur journaliste-écrivain, à l'instar d'un auteur de FASP, fait une analyse minutieuse du milieu professionnel dans lequel il opère, décrit les produits mis sur le marché et analyse l'environnement concurrentiel et réglementaire de Salomon Brothers. Il rapporte, avec un grand sens du suspense et du récit, une multitude de tranches de vie de ses collègues et du milieu de la finance en général. Cette combinaison de deux approches, qui se superposent, confère à ce livre l'intérêt du vécu autobiographique $^{19}$ ainsi que l'ouverture à des enjeux plus larges, caractéristiques de la FASP.

\subsection{L'apport des travaux des sociologues pour aborder la culture professionnelle}

La dimension universelle du terme "culture " a donné lieu à plusieurs centaines de définitions, tant son caractère polysémique relève de champs aussi divers que la sociologie, la psychosociologie ou l'ethnologie. La notion de culture est applicable à des nations, à des régions, ou à une communauté professionnelle et à une organisation, et ce sont ces derniers aspects qui nous intéressent dans cette étude. L'appartenance à un groupe, la constitution progressive de principes communs et de valeurs partagées participent de la culture, concourent à la vie sociale du groupe.

18 S'agissant plus particulièrement de l'entreprise et notamment de la période des années 1980-1990, Geert Hofstede est l'un des premiers anthropologues à s'être intéressé aux phénomènes culturels. Selon Hofstede (2003 : 5), la culture est un phénomène collectif, appris, qui se définit comme «the collective programming of the mind which distinguishes the members of one group or category of people from another $»$.

Au critère social et «mental » qui permet, selon Hofstede, de circonscrire la culture à un groupe ou à une catégorie de personnes spécifiques, Edgar H. Schein (2009: 27) ajoute un axe historique, un critère d'efficacité et une dimension normative :

Culture is a pattern of shared tacit assumptions that was learned by a group as it solved its problems of external adaptation and internal integration, that has worked well enough to be considered valid and, therefore, to be taught to new members as the correct way to perceive, think, and feel in relation to those problems.

20 Si le cadre de référence de notre analyse est le monde professionnel des milieux financiers américains considéré à travers le prisme d'une expérience chez Salomon Brothers, l'examen du phénomène culturel dans son ensemble et de son influence sur les pratiques des professionnels ne saurait faire abstraction des effets multiples des cultures des communautés successives ou parallèles dans lesquelles l'individu a évolué. En effet, il a construit son identité, ses croyances, sa façon d'être et d'interagir dans sa famille et sa classe sociale, dans les pays où il a vécu et les cultures nationales qui leur sont attachées, les écoles et universités ${ }^{20}$ où il s'est formé et, bien entendu, les divers environnements professionnels ${ }^{21}$ dans lesquels il s'est intégré et il a travaillé.

La notion de "programmation mentale" se traduit dans les organisations par des repères intégrés par les employés (symboles, héros de l'entreprise ou de l'industrie) et des pratiques au quotidien ou récurrentes ${ }^{22}$ telles que les rituels (processus codifié voire initiatique de recrutement, de licenciement, annonces individuelles des bonus, annonces publiques de transactions réussies, etc.), reposant sur les valeurs-clés qui 
orientent les actions ou attitudes du groupe, l'ensemble prenant la forme, selon Hofstede, d'un oignon aux couches multiples. Il s'ensuit que les couches superficielles, liées aux pratiques ayant cours dans l'entreprise, sont plus faciles à observer, alors que les valeurs sous-jacentes sont plus difficiles à mettre au jour.

Schein (2009: 21) distingue également plusieurs niveaux constitutifs de la culture organisationnelle :

i. la partie observable, mais difficilement déchiffrable sans éclairage par un insider, qui résulte des choix mis en œuvre en matière de structures (architecture, décor, open space...), de pratiques et de processus et qu'il choisit d'appeler de façon large des artifacts $^{23}$;

ii. les valeurs et principes embrassés par le groupe, son éthique, qui ne correspondent pas toujours aux comportements visibles et nécessitent le cas échéant d'engager une recherche plus approfondie ;

iii. les hypothèses de base, les croyances partagées par le groupe sur lesquelles repose l'ensemble.

Selon Joanna Brewis (2007 : 351), qui s'appuie sur les travaux de Charles Handy (1993), une « culture de pouvoir " peut être observée dans divers milieux, dont ceux du trading et de la finance. Elle est caractérisée par une source centrale de pouvoir (fondateur); une relation de confiance entre le centre (direction) et les acteurs à la périphérie ; un faible nombre de règles et de routines ; une culture forte, marquée par la cohésion et la flexibilité où des individus très "politiques " et disposés à prendre des risques sont à leur avantage ; un environnement de travail dur et compétitif. Il convient cependant de noter que cette culture de pouvoir ${ }^{24}$ est observée par le chercheur dans un contexte d'organisation de taille moyenne (petite société d'investissement ou de trading), dont les propriétés et contraintes peuvent sensiblement différer de celles des grandes institutions de référence.

Les travaux des sociologues sont susceptibles de se heurter à de multiples obstacles. La culture des milieux de la finance est difficile à identifier et plus encore à interpréter pour le chercheur, en raison notamment d'un habitus et d'un référentiel culturel qui créent un fossé quasiment insurmontable entre insiders et outsiders. La difficulté d'accès à une salle de marché (pour des raisons de confidentialité et de sécurité), la langue spécialisée utilisée par les professionnels des marchés, la rapidité des transactions et la méfiance des opérateurs à l'égard des observateurs extérieurs constituent des obstacles redoutables. Ainsi le chercheur Olivier Godechot, membre de l'Association d'études sociales de la finance, reconnaît qu'au terme d'un stage de cinq mois en finance, il s'est heurté au refus de certains traders de remplir, voire de lire, son questionnaire, au motif que l'anonymat ne leur paraissait pas assuré ou que l'enquête pouvait avoir été inspirée par le responsable de la salle des marchés $(2005: 285)$.

Une autre difficulté potentielle pour l'outsider est d'ordre subjectif. Les repères permettant d'interpréter un fait culturel isolé tiennent pour beaucoup à un système, une échelle de localisation, potentiellement dénuée de fondement scientifique, mais enracinée dans la socialisation et dans l'expérience (Beacco 2000 : 127) dont ne dispose pas l'outsider. A fortiori, une telle compétence interprétative présuppose un degré d'intimité qui en limite probablement le champ dans le temps et dans l'espace (Isani $2004: 14)$.

Pour un analyste extérieur au milieu culturel étudié, l'accès aux données autres que médiatisées requiert une approche ethnographique avec présence sur le terrain et observation des professionnels dans leur environnement. En outre, au vu du statut de 
non-spécialiste de l'observateur, les résultats ou interprétations du discours doivent naturellement être corroborés par un spécialiste du domaine (specialist informant). Dans l'étude du genre appliquée à un domaine spécialisé, cette démarche est importante, si l'on souhaite introduire dans l'analyse une explication appropriée plutôt qu'une simple description (Bhatia 1993 : 34).

\section{Méthodologie}

Compte tenu de la difficulté, pour l'outsider qu'est le chercheur en anglais de spécialité, à se faire accepter dans ce milieu professionnel particulier, nous avons choisi de montrer comment un ouvrage comme celui de Michel Lewis peut constituer une source précieuse d'informations et lui apporter matière à enrichir sa connaissance du milieu et de sa culture.

Dans la mesure où nous cherchions à identifier, au fil du récit, les pratiques en cours chez Salomon Brothers, nous nous sommes d'abord livré à une lecture attentive du texte, permettant de repérer un certain nombre d'éléments importants. Notre objectif était de dresser un inventaire des normes, des usages et plus généralement de l'ensemble des marqueurs qui qualifient la culture de cette banque et, dans une certaine mesure, du secteur financier. En effet, ces aspects constituent une sorte de sésame qui conditionne l'intégration des nouveaux membres au milieu de la finance.

Cette première approche bottom up qui part de la source primaire, l'ouvrage lui-même, nous a d'abord permis de relever un certain nombre d'informations relatives aux symboles, aux rites ainsi qu'aux personnages emblématiques décrits dans le texte, en nous inspirant des approches adoptées par G. Hofstede et E. Schein pour identifier les phénomènes culturels au sein de l'entreprise.

L'analyse de ces phénomènes est riche d'enseignements sur les valeurs et les normes en vigueur chez Salomon Brothers. C'est le cas, par exemple, du programme de formation des nouveaux embauchés, décrit en détail dans Liar's Poker, véritable programme d'assimilation sociale ${ }^{25}$, riche en marqueurs explicites de la culture propre à cette grande banque d'affaires. Assis dans l'équivalent d'une salle de classe, les jeunes analystes se voient présenter les départements et activités de Salomon Brothers par des professionnels renommés. Ces présentations au style souvent très direct donnent lieu à des anecdotes diverses, ayant souvent valeur de symbole, dont Lewis se souvient encore deux ans plus tard. Le lecteur apprend ainsi que l'un des intervenants use d'une métaphore ciblée pour capter l'attention de son auditoire en invitant à voir dans Salomon Brothers une jungle ${ }^{26}$ (symbole) dans laquelle sont lâchés tous les stagiaires (rituel) qui devront apprendre à survivre (notamment dans la salle de marché). Le programme, obligatoire, est destiné à transmettre la tradition orale (rituel), au moyen de "war stories» (item symbolique), que racontent ceux qui en ont connu une issue glorieuse (héros). Nous obtenons une typologie qualitative des pratiques et des comportements ayant cours dans la banque et recherchés ou encouragés chez les " Salomonites", en nous appuyant autant que nécessaire sur l'éclairage apporté par l'auteur (explication, métaphore, référence historique, contexte ...).

31 Cette approche bottom up, fondée sur la lecture et par conséquent qualitative, mais peut-être subjective, devait donc être complétée par un traitement statistique objectif de la version numérique de l'ouvrage. Nous souhaitions, en effet, soumettre notre premier bilan des marqueurs représentatifs de la culture d'entreprise relevés 
manuellement dans l'ouvrage à une analyse plus systématique grâce au concordancier Antconc $^{27}$ : il importait de pouvoir mesurer objectivement la fréquence de leurs occurrences, et d'évaluer leur poids relatif. Le concordancier permet aussi de vérifier la pertinence des marqueurs retenus en consultant le contexte immédiat des mots. Une liste des termes clés est également fournie, allant du plus fréquent au moins fréquent. Nous avons ensuite affecté l'occurrence la plus élevée d'un score de 100 et exprimé les scores des autres termes de manière proportionnelle, jusqu'au score de 2 . Trois termes clés à faible fréquence (score de 1) ont été ajoutés manuellement à la liste pour référence ultérieure : symbole, rite et rituel.

Par ailleurs, afin de garder à une entrée la pertinence globale du terme, nous avons regroupé des termes de valence comparable (salesman/salesmen, game/gamble ...) ou encore les différentes universités qui alimentent le programme en jeunes diplômés (Harvard, Stanford, Yale, Princeton). Une version électronique "longue » issue du concordancier et correspondant aux critères de choix (scores 2 à 100) est établie.

Nous avons vérifié par concordancier le contexte de certaines expressions de l'ouvrage, comme celles contenant le terme hour(s) pour identifier la charge de travail (normale en l'espèce) ou une combinaison de mots comme investment bankers pour confirmer son poids relatif, assez élevé (score 16), dans le texte. De même, nous ne retenons que le nombre d'occurrences qui correspond à l'acception à laquelle nous nous intéressons : pour le terme top, seules les occurrences correspondant à une mesure de l'intérêt pour le sommet des organisations ont été retenues, la fréquence passant de 36 à 25 pour cette expression. Les résultats de ces analyses quantitatives sont présentés en annexe.

\section{Résultats et analyse}

L'approche qualitative «bottom up » menée dans un premier temps permet de constater que nous sommes en présence d'une culture professionnelle forte. Sans grande surprise, compte tenu de son importance aux yeux des candidats comme des professionnels de la finance, l'argent apparaît d'emblée dans le livre et est évoqué tout au long du récit. Si l'on se réfère aux marqueurs de culture remarquables retenus par Hofstede dans sa classification, l'argent apparait comme étant un symbole à plus d'un titre : symbole lié à la promesse d'une meilleure qualité de vie, mais aussi preuve pour certains $^{28}$ de leur valeur propre ( $L P: 293$ ). Il n'est pas surprenant que le terme money, avec un total de 306 occurrences, soit ainsi le marqueur culturel le plus fréquent. Dans la banque d'investissement, l'argent a ceci de particulier qu'il constitue à la fois l'objectif de l'activité, un des moyens d'action, et la mesure du résultat. Comme le montre Lewis, la recherche du résultat met la banque entière sous tension. Un objectif prioritaire devient ainsi l'objectif de tout le corps social de la banque, mobilisé dans une démarche collective qui laisse peu de place aux scrupules; on pourrait dans ce cas évoquer une véritable culture du résultat. Devant la pression, il est courant qu'un vendeur soit amené à sacrifier un de ses clients - «blowing up a client» - en lui conseillant des actifs risqués dont la banque souhaite s'alléger.

Plusieurs chapitres du livre portent sur l'intégration des banquiers nouvellement recrutés au sein de l'entreprise et sur la manière dont Salomon Brothers leur transmet sa culture par l'intermédiaire du Training Program. Il s'agit d'une forme d'intégration à marche forcée qui reflète avec une certaine brutalité le concept de «programmation mentale collective " défini par Hofstede. Celle-ci s'effectue sur un terreau réceptif: 
avant même de rejoindre la banque, les candidats voient dans les banquiers d'affaires une sorte de héros, une race à part, celle des maitres en dealmaking (LP: 30); peu d'autres métiers trouvent grâce à leurs yeux. Le concordancier nous permet d'identifier rapidement les vrais héros de l'entreprise, identifiés comme tels par Lewis, qui sont au nombre de trois :

- Lewie Ranieri, qui apparaît au deuxième rang sur notre liste, est le patron du département des obligations hypothécaires, qui fit la notoriété de Salomon à cette époque. C'est, nous dit Lewis, le conquérant, la légende de Salomon, l'incarnation du concept de succès à la Salomon. Modèle de la «culture Salomon ", Ranieri, Italien bon vivant, amateur de pizzas, est aussi un symbole d'ascension sociale fulgurante, de management au plus près des équipes, d'anticipation et d'agressivité commerciale.

- Ron Dipasquale est emblématique du pied de nez fait à la concurrence : trader de seconde zone originaire du back office ${ }^{29}$, il se fait par erreur débaucher à prix d'or par Merrill Lynch. L'erreur coûte deux millions de dollars à Merrill et permet à Dipasquale de revenir en héros reprendre son activité chez Salomon.

- John Gutfreund, le patron de Salomon Brothers, est le héros d'un livre de propagande assez maladroit, édité à la gloire de Salomon et distribué à tous les stagiaires. Héros involontaire de Liar's Poker, il voue à Lewis une rancune tenace ${ }^{30}$.

Il convient également de mentionner John Meriwether, même si l'auteur du livre ne le qualifie pas explicitement de héros; en effet, c'est un trader de renom, membre du conseil de direction de Salomon et très respecté par ses troupes. Il montre ses capacités en matière d'enchères et de bluff lorsque Gutfreund vient le défier pour une partie de poker menteur à un million de dollars.

e recours aux grilles de lecture culturelle de Hofstede et de Schein fait ressortir l'usage de symboles comme celui de la jungle dans laquelle sont lâchés les jeunes diplômés en formation, les bretelles rouge vif, apanage des managing directors, le diplôme d'économie considéré par les recruteurs comme un produit de substitution à un éventuel test de QI. Ces observations confirment l'impression de milieu compétitif, que le taux de sélection (1 admis/60 candidats) pour le programme de recrutement laissait entrevoir. Les marqueurs culturels sur l'ambition professionnelle et sur l'esprit de compétition relevés lors de notre première approche bottom up sont parlants: préparation à l'affrontement, anticipations portant sur les futurs gagnants et perdants, origine universitaire des candidats et vision des parcours d'excellence, etc. Le concordancier permet de valider ces hypothèses, avec par exemple 25 entrées retenues pour le terme top ${ }^{31}$ et 28 pour best ${ }^{32}$.

Lewis voit dans le programme de formation un rite d'initiation, qui repose selon nous sur deux approches conjuguées. D'une part, le mimétisme professionnel imposé aux recrues est pensé, structuré comme un programme d'assimilation sociale, mais aussi distillé, plus ou moins subtilement, sous forme d'activités de groupe, de formation par l'exemple, d'encadrement par un mentor ( $a$ rabbi»), de relégation non dite des départements les moins prestigieux, frappés d'ostracisme et, enfin, d'apprentissage de la terminologie professionnelle interne. D'autre part, dans le cadre d'une épreuve darwinienne, les jeunes recrues sont envoyées en salle de marché. Ce rite de passage est douloureux pour l'ego. Les stagiaires subissent un système destiné à tester leur capacité d'adaptation et à leur faire prendre conscience de leur position d'infériorité : ils doivent être physiquement présents dans la salle de marché, tout en n'ayant pas de tâches précises ni de poste dédié où s'installer, de sorte qu'ils cherchent sans succès 
une contenance, conscients de n'être pas à leur place : « the firm built a system around the belief that trainees should wriggle and squirm » (LP: 47). Dans la salle de marché, véritable champ de mines ( $L P: 57)$, ils sont ainsi contraints de jouer le rôle de figurants invisibles, sans droit de parole, que les professionnels peuvent humilier à loisir (LP: 58). Les rares femmes ne sont en rien épargnées dans cet environnement masculin et brutal $^{33}$. Nous retrouvons le terme que Lewis n'emploie qu'une fois pour résumer cette expérience d'endoctrinement qu'il a subie: brainwashing (LP: 55). L'auteur prend cependant ses distances avec le programme d'adhésion à la "culture Salomon » en précisant que, sur le long terme, ce lavage de cerveau n'obtient pas l'effet escompté en matière de fidélisation des professionnels.

Une fois le programme de formation achevé, les jeunes diplômés rejoignent leur structure de rattachement (par exemple desk de trading ou équipe de vente). Ils découvrent un autre volet de la culture, celui d'une structure hiérarchique relativement plate, peu contraignante une fois les usages intégrés, constituée de cercles concentriques d'appartenance (équipe ou desk, département de trading et enfin Salomon Brothers). L'instinct du trader le conduit en effet d'abord à privilégier sa position au sein du desk. Sa loyauté suit une relation d'ordre établie, «Desk, Firm, God, Country ", selon la formule proposée par Robert Wosnitzer (2014:1), professeur à la Stern School of Business et ancien opérateur de marché pour Lehman Brothers. D'autres rituels viennent par la suite se substituer au programme de formation: ceux de l'attribution des bonus, les mauvaises blagues faites aux collègues et les sorties en équipe pour un dîner ou pour jouer au casino.

Enfin, nous observons que la période des années 1980 est celle d'une évolution de la culture de Salomon Brothers, banque qui comportait de nombreux professionnels d'origine juive et qui diversifie ses recrutements en direction des WASP (blancs, anglosaxons, protestants). Salomon cherche par ailleurs à attirer des diplômés (de préférence titulaires d'un MBA ou d'un doctorat) des meilleures universités ${ }^{34}$ afin de s'adapter aux exigences de l'innovation financière et de la finance quantitative. La culture du trader «street-smart » cède la place à celle du professionnel, qui valorise son expertise dans l'analyse et l'évaluation du risque.

41 Le tableau qui se dessine à la lumière de cette étude de la culture des milieux financiers est complexe. Sommes-nous en présence d'une monoculture de l'argent, obsessionnelle, voire addictive, comme le reconnaît l'ancien trader Sam Polk (2014) dans le New York Times, ou d'une culture à plusieurs facettes? Derrière la dominante argent se profilent en effet des cultures du risque, du résultat et de la performance (au sens compétitif) et sans doute une composante orientée vers le pouvoir. D. Collins s'intéresse quant à lui au thème de l'accession au pouvoir et à ses attributs par le biais d'une analyse de Liar's Poker inspirée par Le Prince de Machiavel, mais il ne précise pas pourquoi les managers de Salomon ne cherchent pas à obtenir et à garder un " pouvoir permanent» (1992: 372). Il semble que le pouvoir des professionnels confirmés de Salomon Brothers, les big swinging di...s soit surtout lié à leur statut, qui leur confère une forte capacité de nuisance dont pâtissent les jeunes embauchés, et une autorité avérée que subissent les vendeurs les moins aguerris ${ }^{35}$. Lewis et son ancien patron s'accordent, cependant, pour considérer que ce dernier n'avait que peu de moyens de contrôle sur ses subordonnés. Si la question de l'argent est centrale, elle dérive mécaniquement vers la recherche du résultat et les moyens d'y parvenir. La réponse est peut-être à 
rechercher dans les causes économiques structurelles du tournant des dernières décennies.

Le sujet mériterait une analyse plus approfondie, mais plusieurs facteurs ont concouru à l'apparition d'une culture du risque, au service de la recherche du résultat. Les ouvertures nées de la dérégulation ont mis au jour la nécessité de recourir à la technologie pour une exploitation à grande échelle. Les banques commerciales, mieux armées financièrement, ont exercé une pression concurrentielle forte sur le secteur, contraignant des banques d'investissement (comme Salomon Brothers) à abandonner la structure partenariale pour se mettre en quête de financements et à s'orienter vers des niches moins concurrentielles mais plus risquées (par exemple, trading pour compte propre). La recherche du profit, conjuguée à la tension concurrentielle, a abouti à une situation très rémunératrice pour les opérateurs de marché spécialisés, mais aussi risquée pour les institutions. Salomon, comme plusieurs de ses concurrents, s'est ainsi engagé dans des opérations douteuses, voire illégales.

Le changement de paradigme imposé par la crise de 2008 soulève la question de la validité du modèle culturel des institutions financières, critiqué par les médias et par le grand public. Quand Lloyd Blankfein, patron de la banque Goldman Sachs, déclare en 2009 «[I am] doing God's work», le journaliste John Carney (2009) voit une provocation qui laisse peu de place à une réconciliation avec un public en colère. Aux États-Unis, la loi Dodd-Frank de 2010 encadre désormais très strictement les activités des banques d'investissement et comporte plusieurs dispositions relatives à la transparence applicable aux rémunérations des dirigeants. Les attraits d'une culture «à la Salomon Brothers » - rémunération très élevée, mais aussi promesse de risque et de frisson ${ }^{36}$ - se sont a priori dissipés. Aux yeux des diplômés de business schools américaines, la banque d'investissement classique semble perdre de son attractivité ${ }^{37}$ face à des secteurs plus porteurs ou moins réglementés mais rémunérateurs, tels que les fonds d'investissement en sociétés non cotées, la haute technologie appliquée à la finance (la Fintech) ou le conseil en fusions-acquisitions ou en stratégie. Martin Wolf $(2010: 228)$, journaliste au Financial Times, revient sur les critiques formulées à propos du système de rémunération utilisé dans les institutions financières. Selon lui, le niveau de rétribution ne doit pas être régulé et relève de la seule fiscalité. Il est en revanche favorable à une structure de paie qui rend les banquiers responsables, par des mesures telles que le versement conditionnel et différé ${ }^{38}$, voire remboursable, d'une partie de la rémunération ou l'impossibilité d'obtenir un nouvel emploi dans le secteur pour les managers ayant pris des risques manifestement excessifs.

Pour de nombreux experts, la réponse réglementaire est un levier qui doit être complété par une remise en cause interne de la culture des banques. Selon un cabinet de conseil en stratégie qui s'interroge sur les transformations qui attendent le secteur de la finance (Daruvala et al. 2012 : 39-40), les banques sont appelées à influer sur les mentalités de leurs employés. Il en va de la responsabilité des dirigeants : les banquiers les plus gradés devraient voir la transformation culturelle à mener en interne comme une question stratégique et non plus comme un simple problème de relations publiques.

La question de la culture à l'œuvre dans le milieu bancaire est aussi devenue cruciale aux yeux des banques centrales. M. Carney (2013), ancien de Goldman Sachs, puis gouverneur de la Banque du Canada jusqu'en 2013 et actuellement gouverneur de la Banque d'Angleterre, relève que «[...] plus que maîtriser la valorisation des options, 
l'évaluation des entreprises ou la comptabilité, vivre selon des valeurs justes sera le défi le plus important pour [...] les étudiants qui vont travailler en finance chaque année ${ }^{39}$. Très récemment, la revue American Banker mentionnait une déclaration de Janet Yellen, présidente de la Réserve fédérale, soulignant que les banques avaient encore des progrès à faire en matière de culture interne $e^{40}$.

\section{Conclusion}

$\mathrm{Au}$ terme de cette étude, il apparaît que Liar's Poker forme une combinaison particulièrement attractive pour le chercheur en anglais de spécialité. Son auteur - qui publie régulièrement articles et ouvrages représentatifs de l'actualité économique sait parfaitement démonter la mécanique des marchés et des grandes institutions financières pour en présenter l'histoire, les protagonistes et bien entendu les enjeux. Toutefois, tout en prenant en compte les particularités du milieu professionnel de la finance, il reste attentif au facteur humain, à la psychologie des acteurs qui influe sur leurs discours et sur leurs actions quand il décrit la partie de bluff entre le président et un trader, la compétition et les jeux de pouvoir entre les patrons d'équipe, les états d'âme des candidats à l'embauche dans les banques d'affaires, l'obsession des recrues pour la carrière professionnelle optimale hors de laquelle il n'est point de salut, ou encore les dilemmes éthiques des professionnels.

Le charme ludique du livre peut être, paradoxalement, une source de difficulté, car l'auteur joue avec ses personnages, force le trait pour déclencher le rire, au point que l'on ne distingue pas toujours la vérité de l'histoire racontée. Il n'en reste pas moins que cet ouvrage très documenté offre une source d'informations intéressante au chercheur en anglais de spécialité pour appréhender la culture des milieux financiers, dans un contexte de cycles économiques qui voit ressurgir aujourd'hui encore le débat du risque, de sa rémunération, ainsi que de la place de la finance dans l'économie.

\section{BIBLIOGRAPHIE}

ABOLAFIA, Mitchel. 2001 [1996]. Making Markets: Opportunism and Restraint on Wall Street. Cambridge, MA : Harvard University Press.

BEACCO, Jean-Claude. 2000. Les dimensions culturelles des enseignements de langue. Paris : Hachette.

BHATIA, Vijay K. 1993. Analysing Genre: Language Use in Professional Settings. Londres : Longman.

BREWIS, Joanna. 2007. « Culture ». In Knights, David \& Hugh Willmott (dir.), Introducing Organizational Behaviour and Management. Londres : Thomson Learning , 344-374.

BRUCK, Connie. 1988. The Predators' Ball. New York : Simon \& Schuster.

BURROUGH, Bryan \& John HELYAR. 1990. Barbarians at the Gate. New York : Harper \& Row. 
CARNEY, John. 2009. « Lloyd Blankfein says he is doing 'God's work' ». Business Insider, consulté le 21 juin 2016 < http://www.businessinsider.com/lloyd-blankfein-says-he-is-doing-godswork-2009-11?IR=T>.

CARNEY, Mark. 2013. Remarks by Mark Carney, Governor of the Bank of Canada, 7th Annual Thomas d'Aquino Lecture on Leadership, Richard Ivey School of Business, consulté le 19 juin 2016 <http://www.bankofcanada.ca/wp-content/uploads/2013/02/remarks-250213.pdf>.

CetinA, Karin K. \& Alex PREDA (dir.). 2005. The Sociology of Financial Markets. Oxford : Oxford University Press.

CHAPPE, Raphaële, Edward NeLl \& Willi SEMMLER. 2012. « On the history of the U.S. financial culture ». Geschichte Und Gesellschaft. Sonderheft 24, 59-84.

ColuINS, Denis. 1992. « An ethical analysis of organizational power at Salomon Brothers ». Business Ethics Quarterly 2, 367-377.

DarUVAla, Toos, Miklos DiETZ, Philipp HARLE, Joydeep senGUPTA, Matthias voelKel \& Eckart WINDHAGEN. 2012. «The triple transformation: Achieving a sustainable Business model ». $2^{\text {nd }}$ McKinsey Annual Review on the banking industry, consulté le 21 juin $2016<\mathrm{http}$ :// www.mckinsey.com/industries/financial-services/our-insights/the-search-for-a-sustainablebanking-model>.

GODECHOT, Olivier. 2005. Les traders : essai de sociologie des marchés financiers. Paris : La Découverte.

GodiWALLA, Nina. 2011. Suits: A Woman on Wall Street. New York : Atlas.

« Goldman Sachs Sweetens Deal for Young Bankers ». 2015. Wall Street Journal, consulté le 9 décembre 2016 <http://www.wsj.com/articles/goldman-sachs-brings-back-junior-bankerprogram-1446753898>.

HANDY, Charles. 1993. Understanding Organizations: Managing Differentiation and Integration. New York : Oxford University Press.

HoFSTEDE, Geert. 2003. Cultures and Organizations. Londres : Profile Books Ltd.

ISANI, Shaeda. 2004. «Compétence de culture professionnelle : définition, degrés et didactisation ». ASp 43-44, 5-21.

KIRKLAND, Richard, Jr. 1989. « Animal House Goes to Wall Street ». Fortune Magazine, consulté le 10 décembre 2016 <http://archive.fortune.com/magazines/fortune/fortune_archive/ 1989/11/20/72762/index.htm>.

LEWIS, Michael. 1991. The Money Culture. New York : W.W. Norton \& Co.

LEWIS, Michael. 1992. Pacific Rift. New York : W.W. Norton \& Co.

LEWIS, Michael. 1997. Losers [Trail Fever]. New York : Random House.

LEWIS, Michael. 1999. The New New Thing. Londres : Hodder and Stoughton.

LEWIS, Michael. 2001. Next: The Future Just Happened. New York : W.W. Norton \& Co.

LEWIS, Michael. 2003. Moneyball. New York : W.W. Norton \& Co.

LEWIS, Michael. 2005. Coach: Lessons on the Game of Life. New York : W.W. Norton \& Co.

LEWIS, Michael. 2006 [1989]. Liar's Poker. Londres : Hodder and Stoughton.

LEWIS, Michael. 2006. The Blind Side. New York : W.W. Norton \& Co. 
LEWIS, Michael. 2008. « The End: How Wall Street did itself in ». Conde Nast Portfolio (archives du 11 novembre 2008). <upstart.bizjournals.com/news-markets/national-news/portfolio /2008/11/11/ the-end-of-wall-streets-boom.html?page=all> .

LEWIS, Michael. 2009. Panic. New York : W.W. Norton \& Co.

LEWIS, Michael. 2009. Home Game. New York : W.W. Norton \& Co.

LEWIS, Michael. 2010. The Big Short. New York : W.W. Norton \& Co.

LEWIS, Michael. 2011. Boomerang. New York : W.W. Norton \& Co.

LEWIS, Michael. 2014. Flash Boys. New York : W.W. Norton \& Co.

LEWIS, Michael. 2016. The Undoing Project. New York : W.W. Norton \& Co.

O'SULLIVAN, Mary. 2013. « Celebrating youth: Historical origins of the U.S. stock market's appetite for novelty ». In Dosi, G. \& L. Galambos (dir.), The Third Industrial Revolution in Global Business. New York : Cambridge University Press, 288-291.

PETIT, Michel. 2000. « Le paratexte dans la fiction à substrat professionnel ». Bulletin de la société de stylistique anglaise 21, « Texte et paratexte ». Actes du Colloque de Nanterre 4-5 juin 1999, 173-195.

PoLK, Sam. 2014. « For the Love of Money ». The New York Times Sunday Review. Mis en ligne le 18 janvier 2014, consulté le 29 juin 2016 <http://www.nytimes.com/2014/01/19/ opinion/sunday/ for-the-love-of-money.html?_r=0>.

RESCHE, Catherine. 2005. «Les mission statements des grandes entreprises cotées en Bourse : prélude à l'étude d'un genre particulier de textes dictés par plusieurs contextes ». ASp 47-48, 530.

Rolfe, John \& Peter Troob. 2001. Monkey Business. New York : Hachette Group.

SCHEIN, Edgar H. 2009. The Corporate Culture Survival Guide. San Francisco, CA : Jossey-Bass.

STERN, Richard. 1989. «Book \& Business: Overpaid and Guilt-Ridden ». The New York Times, consulté le 09 décembre 2016 <www.nytimes.com/1989/10/29/books/book-business-overpaidand-guilt-ridden.html>.

STEWART, James. 1991. Den of Thieves. New York : Simon \& Schuster.

VAN DER YeUGHT, Michel. 2009. Une histoire de Wall Street. Paris : Eska.

VAN DER YEUGHT, Michel. 2012. L'anglais de la bourse et de la finance : description et recherche.

Paris : Ophrys.

WoLF, Martin. 2010. « Why and how should we regulate pay in the financial sector? ». In Turner, A., A. Haldane, P. Woolley, S. Wadhwani, C. Goodhart, A. Smithers, A. Large, J. Kay, M. Wolf, P. Boone, S. Johnson \& R. Layard (dir.), The Future of Finance. Londres : The London School of Economics and Political Science, 227-237.

WoLfE, Tom. 1987. The Bonfire of the Vanities. New York : Farrar, Straus and Giroux. WOSNITZER, Robert. 2014. « Desk, Firm, God, Country: Proprietary trading and the speculative ethos of financialism ». Monographie inédite, New York University. <http:// search.proquest.com/docview/1553234548?accountid=42654>. 


\section{ANNEXES}

Liste des 100 premiers termes relatifs aux symboles, rites et rituels, héros et autres observations

\begin{tabular}{|c|c|c|c|}
\hline Terme (total : 9013 termes) & Fréquence absolue & Score & Rang \\
\hline Money & 306 & 100 & 1 \\
\hline Ranieri & 227 & 74 & 2 \\
\hline Gutfreund & 222 & 73 & 3 \\
\hline dollars & 203 & 66 & 4 \\
\hline training, trainee(s) & 197 & 64 & 5 \\
\hline department & 182 & 59 & 6 \\
\hline customer(s),client(s) & 160 & 52 & 7 \\
\hline million & 157 & 51 & 8 \\
\hline management, manager(s) & 132 & 43 & 9 \\
\hline salesman/men & 122 & 40 & 10 \\
\hline new & 122 & 40 & 11 \\
\hline customers, client & 92 & 30 & 12 \\
\hline Milken & 90 & 29 & 13 \\
\hline sales, selling & 71 & 23 & 14 \\
\hline program & 67 & 22 & 15 \\
\hline Rubin & 67 & 22 & 16 \\
\hline stock & 65 & 21 & 17 \\
\hline took & 65 & 21 & 18 \\
\hline want & 65 & 21 & 19 \\
\hline markets & 64 & 21 & 20 \\
\hline men & 64 & 21 & 21 \\
\hline profits, profitable & 60 & 20 & 22 \\
\hline Drexel & 52 & 17 & 23 \\
\hline
\end{tabular}




\begin{tabular}{|c|c|c|c|}
\hline f..k & 51 & 17 & 24 \\
\hline Strauss & 49 & 16 & 25 \\
\hline game, gamble & 49 & 16 & 26 \\
\hline investment banker(s) & 48 & 16 & 27 \\
\hline risk & 47 & 15 & 28 \\
\hline desk & 44 & 14 & 29 \\
\hline Mortara & 42 & 14 & 30 \\
\hline HSYP (Harvard, Stanford, etc.) & 42 & 14 & 31 \\
\hline pay & 41 & 13 & 32 \\
\hline Grady & 39 & 13 & 33 \\
\hline Merrill & 39 & 13 & 34 \\
\hline play, played & 39 & 13 & 35 \\
\hline boss & 37 & 12 & 36 \\
\hline fired & 36 & 12 & 37 \\
\hline Perelman & 36 & 12 & 38 \\
\hline create & 36 & 12 & 39 \\
\hline learn & 34 & 11 & 40 \\
\hline bonus & 33 & 11 & 41 \\
\hline jungle & 33 & 11 & 42 \\
\hline Meriwether & 32 & 10 & 44 \\
\hline opportunist & 33 & 11 & 43 \\
\hline geek & 31 & 10 & 45 \\
\hline women & 29 & 9 & 46 \\
\hline best & 28 & 9 & 47 \\
\hline economics & 27 & 9 & 48 \\
\hline idea & 26 & 8 & 49 \\
\hline family & 26 & 8 & 50 \\
\hline
\end{tabular}




\begin{tabular}{|c|c|c|c|}
\hline speaker & 26 & 8 & 51 \\
\hline top & 25 & 8 & 52 \\
\hline career & 25 & 8 & 53 \\
\hline member & 25 & 8 & 54 \\
\hline self & 25 & 8 & 55 \\
\hline control & 24 & 8 & 56 \\
\hline poker & 24 & 8 & 57 \\
\hline value & 24 & 8 & 58 \\
\hline group & 23 & 8 & 59 \\
\hline Liar & 22 & 7 & 60 \\
\hline power & 22 & 7 & 61 \\
\hline better (competitive comparison) & 21 & 7 & 62 \\
\hline d.ck & 21 & 7 & 63 \\
\hline piranha & 21 & 7 & 64 \\
\hline rich & 21 & 7 & 65 \\
\hline story & 21 & 7 & 66 \\
\hline success & 21 & 7 & 67 \\
\hline team & 21 & 7 & 68 \\
\hline interview & 20 & 7 & 69 \\
\hline shouted & 20 & 7 & 70 \\
\hline hours & 19 & 6 & 71 \\
\hline suits & 19 & 6 & 72 \\
\hline bets & 19 & 6 & 73 \\
\hline Buffett & 19 & 6 & 74 \\
\hline cash & 19 & 6 & 75 \\
\hline culture & 18 & 6 & 76 \\
\hline banker(s) & 17 & 6 & 77 \\
\hline
\end{tabular}




\begin{tabular}{|c|c|c|c|}
\hline change & 16 & 5 & 78 \\
\hline trust & 16 & 5 & 79 \\
\hline deals & 15 & 5 & 80 \\
\hline opportunity & 15 & 5 & 81 \\
\hline shout & 15 & 5 & 82 \\
\hline fear, afraid & 15 & 5 & 83 \\
\hline lunch & 14 & 5 & 84 \\
\hline research & 14 & 5 & 85 \\
\hline trades & 14 & 5 & 86 \\
\hline war & 14 & 5 & 87 \\
\hline phones & 13 & 4 & 88 \\
\hline compensation & 13 & 4 & 89 \\
\hline Japan & 13 & 4 & 90 \\
\hline played & 13 & 4 & 91 \\
\hline corporate finance & 12 & 4 & 92 \\
\hline fortune & 12 & 4 & 93 \\
\hline loyalty & 12 & 4 & 94 \\
\hline ambition & 11 & 4 & 95 \\
\hline casino & 11 & 4 & 96 \\
\hline $\mathrm{cmo}$ & 11 & 4 & 97 \\
\hline win & 11 & 4 & 98 \\
\hline cigar(s) & 11 & 4 & 99 \\
\hline few & 10 & 3 & 100 \\
\hline
\end{tabular}

\section{NOTES}

1. On se reportera utilement à l'ouvrage de Michel Van der Yeught (2012) pour une présentation historique et une analyse détaillée de l'anglais de la bourse et de la finance.

2. Dorénavant, Liar's Poker ou LP.

3. Créée en 1910, Salomon Brothers était une célèbre banque d'investissement américaine prototypique de la finance anglo-saxonne des années 1980 et 1990. 
4. Michael Lewis dispose de son propre site web à l'intention de ses lecteurs, de la presse et de ses clients : <http://michaellewiswrites.com/index.html\#top>.

5. Nous pensons, par exemple, à la titrisation d'emprunts hypothécaires.

6. Selon le critique littéraire du New York Times (Stern 1989), "[Michael Lewis] is obviously as good a writer as he was a bond salesman".

7. À l'issue de leur Graduate Program de deux ans, de trop nombreux jeunes analystes quittaient Goldman Sachs pour monnayer leur expérience chez un concurrent (Wall Street Journal, 5 novembre 2015).

8. À titre d'exemple, nous pouvons mentionner Boston College (Life and Career Planning) et Northwestern University (Kellogg School of Management, cours de finance).

<http://www.bc.edu/schools/cas/capstone/seminars/un505.html>, consulté le 10 décembre 2016.

<http://www.kellogg.northwestern.edu/faculty/papanikolaou/htm/finc460/ln/syllabus_all.pdf>, consulté le 10 décembre 2016.

9. Forums du type Wall Street Oasis, MBA Crystal Ball, Master Admissions, Prépa HEC.

10. Suits (Godiwalla 2011) et Monkey Business (Rolfe \& Troob 2001) détaillent l'expérience de jeunes recrues dans des équipes de corporate finance (chargées de mener des fusions-acquisitions ou des introductions en bourse).

11. Les banques anglo-saxonnes regroupent ces métiers sous une seule appellation, S\&T, pour sales and trading.

12. On retiendra, par exemple, les modèles de Fama sur l'efficience des marchés ou la formule de Black et Scholes appliquée aux options.

13. Cet entretien, rapporté sur le site de Institutional Real Estate, Inc., était aussi une occasion pour Lewis de promouvoir son livre The Big Short. <http://www.irei.com/blog/starring-christianbale-steve-carell-ryan-gosling-abd-brad-pitt/>, consulté le 11 décembre 2016.

14. Il a toutefois effectué un travail minutieux d'investigation pour les écrire.

15. Le terme, traduit par «obligations pourries", désigne des titres obligataires à haut rendement et particulièrement risqués.

16. Discours d'Ivan Boesky devant les étudiants de la Business school de Berkeley en 1986.

17. Par exemple The Bonfire of the Vanities ou The Predators' Ball.

18. "The funniest book about Wall Street I have ever read".

19. Lewis rapporte qu'il a reçu des courriers d'étudiants qui voyaient en Liar's Poker un vademecum de la finance de marché.

20. Lewis est diplômé de Princeton et de la London School of Economics (LSE), deux établissements cibles des recruteurs des grandes banques d'affaires.

21. On peut citer les industries ou services, les organisations, départements, équipes, fonctions occupées, associations, etc.

22. Schein évoque à cet égard la conception courante mais simplificatrice du "the way we do things around here ».

23. Les domaines concernés comprennent en effet aussi bien le code vestimentaire que le degré de formalisme dans les échanges, les modes de prise de décision et de résolution des conflits, la façon d'apprendre, les horaires de travail, etc. (2009: 84).

24. Hofstede s'est intéressé dans les années 1970 à l'acceptation par les membres les moins puissants (d'une nation ou d'un groupe) d'une répartition inégale du pouvoir, mesurée par le Power Distance Index (PDI).

25. "The powers of Salomon Brothers relied on the training programme to make us more like them» (LP: $54)$.

26. « Ya gotta tink a Salomon Bruddahs as like a jungle » (LP : 44) [sic].

27. Le concordancier est en accès libre sur le site < http://www.laurenceanthony.net/ software.html>. 
28. Michael Lewis s'en défend personnellement.

29. Le back office est une activité peu prestigieuse qui consiste, entre autres, à vérifier/enregistrer les transactions passées en salle des marchés, alors que le front office regroupe les activités pratiquées directement au contact du marché et des clients (grandes entreprises, investisseurs institutionnels, etc.), génératrices de profit et sensiblement mieux rémunérées.

30. Une dizaine d'années avant sa mort, Gutfreund accepte de déjeuner avec Lewis et, en fin de repas, conclut laconiquement : "Your fucking book destroyed my career, and it made yours " (Lewis $2008: 6$ ).

31. Exemple d'entrée retenue pour top : « I would one day join them at the top ».

32. Best est, selon le cas, associé à l'entreprise, à un département, aux professionnels concernés (businessmen, traders, salesmen, producers, risk takers, analysts).

33. At Salomon, men traded, women sold ( $L P: 78$ ).

34. Le tri par concordancier fait ressortir les noms des écoles de Harvard, Stanford, Wharton, Yale et Princeton.

35. «Human Piranha », un vendeur doté d'une grande expertise technique, inspire respect voire inquiétude aux traders.

36. Lewis mentionne dans l'introduction de The Money Culture (1991) le célèbre commentaire (également utilisé par Donald Trump) "The money was important, but mainly as a way of keeping score ", avant de préciser " The appeal of [finance] to a young person was its promise of drama ».

37. La proportion de diplômés du MBA de Wharton choisissant un travail en banque d'investissement proprement dite est passée de $26 \%$ environ à la fin des années 1990 à $14 \%$ en moyenne 15 ans plus tard. <https://statistics.mbacareers.wharton.upenn.edu/full-time/>, consulté le 14 janvier 2017.

38. Dispositions mises en œuvre depuis la crise dans de nombreux établissements américains et européens.

39. Traduction effectuée par l'auteur.

40. < http://www.americanbanker.com/news/law-regulation/yellen-warns-on-bank-culturedefends-dodd-frank-progress-1092868-1.html>, consulté le 15 décembre 2016.

\section{RÉSUMÉS}

Cette étude a pour objet de souligner l'intérêt que peut présenter, pour la recherche en anglais de spécialité, l'analyse d'un récit à mi-chemin entre la FASP et une autobiographie. L'analyse a pour support Liar's Poker (1989), dont l'auteur, Michael Lewis, a travaillé pour Salomon Brothers à New York et à Londres en tant que vendeur d'obligations dans les années 1980. Son témoignage a été analysé dans le but de rechercher les traits caractéristiques des pratiques des professionnels de la finance de l'époque, à une période de forte financiarisation des économies occidentales. Les hypothèses qu'une lecture cursive a permis de formuler sur la nature de ces comportements ont été vérifiées à l'aide d'un concordancier. Les résultats mettent en relief l'impact que la période des années 1980 et la culture aux multiples facettes qui lui est associée ont pu avoir sur la réputation des milieux de la finance dans l'opinion publique et l'importance, pour les organisations, d'une gestion dynamique de leur culture. Liar's Poker permet ainsi une meilleure appréhension des usages et pratiques d'un milieu professionnel pour le chercheur comme pour son public d'étudiants, futurs professionnels. 
This article highlights the potential benefits to be derived for ESP research from the study of a narrative which lies halfway between FASP and an autobiography. The study focuses on Liar's Poker (1989), a story whose author, Michael Lewis, worked as a bond salesman in the New York and London offices of Salomon Brothers in the 1980s. His literary testimony is analyzed with a view to identifying the key practices prevailing in the financial milieu of the 1980s, at a time of intensive financialization of Western economies. A study of the book brought to light a number of hypotheses regarding the nature of this culture. The hypotheses were subsequently tested with the use of a concordancer. The analysis highlights the impact which the 1980s and the related multi-faceted culture have had on the reputation of the financial community with the general public, and the need for financial institutions to actively manage their organizational culture. On the whole, Liar's Poker allows for a better understanding of the customs and practices associated with a professional community for both ESP researchers and their student audience of professionals in the making.

\section{INDEX}

Mots-clés : biographie, culture professionnelle, milieu de la finance, récit, Liar's Poker, Wall Street

Keywords : biography, financial community, Liar's Poker, professional culture, narrative, Wall Street

\section{AUTEUR}

\section{MARC ELINE}

Marc Éline est maître de conférences en anglais économique et financier à l'Université Panthéon Assas - Paris 2. Il s'intéresse au management et au milieu bancaire. Il a travaillé pendant plus de vingt ans dans les domaines de la finance et des relations économiques internationales.

marc.eline@free.fr 